Pacific Journal of Mathematics

ON POLYNOMIAL GENERATORS IN THE ALGEBRA OF
COMPLEX FUNCTIONS ON A COMPACT SPACE 


\title{
ON POLYNOMIAL GENERATORS IN THE ALGEBRA OF COMPLEX FUNCTIONS ON A COMPACT SPACE
}

\author{
GABRIEL KatZ
}

In this paper we prove that in the space of all continuous mappings of a $k$-dimensional compact space $X$ into complex linear space $C^{n}$ the imbeddings $F: X \rightarrow C^{n}$ with the property "any complex continuous function on $F(X)$ can be uniformly approximated by complex polynomials on $C^{n}$ " form a dense subset of type $G_{\delta}$, provided that $k \leq \frac{2}{3} n$.

If is known [2] that if the algebra of continuous complex functions $C(X)$ for a topological space $X$ has $k$ multiplicative generators then $X$ has to be acyclic (with complex coefficients) in dimensions $\geq k$. In particular, $C\left(M^{k}\right)$ has at least $k+1$ generators for any closed orientable $k$-manifold $M$. On the other hand, it was proved in [6] that there exist $k+1$ polynomial generators in the algebra $C\left(X^{k}\right)$ for a finite $k$-dimensional simplicial polyhedron $X^{k}$. This means that any such function on $X^{k}$ may be uniformly approximated by complex polynomials in certain specially constructed functions $f_{0}^{*}, \ldots, f_{k}^{*} \in C\left(X^{k}\right)$. In other words, there exists a continuous embedding $F^{*}: X^{k} \rightarrow \mathbf{C}^{k+1}$ of the polyhedron $X^{k}$ into complex vector space $\mathbf{C}^{k+1}$ such that any continuous complex valued function on the image $F^{*}\left(X^{k}\right)$ may be approximated by complex polynomials in the coordinate functions $z_{i}: \mathbf{C}^{k+1} \rightarrow \mathbf{C}, 0 \leq i \leq k$.

It seems that analogous results follow for any compact space $X^{k}$ (not only for polyhedra). Moreover, it is quite natural to conjecture that for $X^{k}$ compact the existence of polynomial approximation on $F\left(X^{k}\right) \subset \mathbf{C}^{k+1}$ is a "general position" phenomenon with respect to perturbations of $F$ : $X^{k} \rightarrow \mathbf{C}^{k+1}$. Note, that this would be a complete complex analog of the classical Whitney theorems [9] (see also [4]).

In this paper we prove similar propositions for imbeddings $F: X^{k} \rightarrow$ $\mathbf{C}^{n}$ satisfying the dimensional condition $k \leq \frac{2}{3} n$. In particular, for 2-dimensional compact spaces $X^{2}$ one has the following result ("complex Whitney theorem"): there are 3 multiplicative generators in the algebra $C\left(X^{2}\right)$, in fact, starting with any $f_{1}, f_{2}, f_{3} \in C\left(X^{2}\right)$ one can perturb them by an arbitrarily small amount to get a set of multiplicative generators for $C\left(X^{2}\right)$. Note, that this is the best possible general result for $k=2$. 
Our main result is

Theorem A. Let $3 k \leq 2 n$. In the space $\operatorname{Map}\left(X^{k}, \mathbf{C}^{n}\right)$ of all continuous mappings of a $k$-dimensional compact space $X^{k}$ into complex linear space $\mathbf{C}^{n}$ consider the mappings $F: X^{k} \rightarrow \mathbf{C}^{n}$ satisfying the following properties:

1. $F$ is an imbedding;

2. any continuous function on $X^{k}$ may be approximated by complex polynomials in the multiplicative generators $f_{1}=z_{1} \circ F, \ldots, f_{n}=z_{n} \circ F$, where $z_{1}, \ldots, z_{n}$ are complex coordinate functions on $\mathbf{C}^{n}$;

3. in particular, $F\left(X^{k}\right)$ is polynomially convex in $\mathbf{C}^{n}$.

These mappings form a dense subset of type $G_{\delta}$ in $\operatorname{Map}\left(X^{k}, \mathbf{C}^{n}\right)$.

The proof of this theorem is based on the following proposition.

THEOREM B. Let $3 k \leq 2 n$. In the space $\operatorname{SL~Map~}\left(Y^{k}, \mathbf{C}^{n}\right)$ of simplicially linear mappings of a finite $k$-dimensional simplicial polyhedron $Y^{k}$ into $\mathbf{C}^{n}$ there exists an open and everywhere dense subset of imbeddings $F: Y^{k} \rightarrow \mathbf{C}^{n}$ such that any continuous function on the image $F\left(Y^{k}\right)$ may be approximated by complex polynomials over $\mathbf{C}^{n}$ and, consequently, $F\left(Y^{k}\right)$ is polynomially convex in $\mathbf{C}^{n}$.

We don't know if Theorems A and B have immediate analogs for smooth regular imbeddings. For example, it is easy to show that there is no smooth regular imbedding $F: \mathbf{C} P^{2} \rightarrow \mathbf{C}^{6}$ of complex projective space $\mathbf{C} P^{2}$ with the tangent bundle of $F\left(\mathbf{C} P^{2}\right)$ being a totally real subbundle of a trivial complex 6-dimensional bundle. On the other hand, $3 \cdot \operatorname{dim} \mathbf{C} P^{2}$ $\leq 2 \cdot 6$, which is perfectly consistent with the dimensional assumptions of Theorems A and B.

Prior to the proof of Theorem B we need to introduce some terminology and to prove some auxiliary propositions.

Let $\mathscr{L}$ be any finite family of real affine subspaces $\left\{V_{\alpha}\right\}_{\alpha \in \mathscr{L}}$ of $\mathbf{C}^{n}$ with the property $V_{\alpha} \nsubseteq V_{\beta}$ for any pair $\alpha, \beta \in \mathscr{L}, \alpha \neq \beta$. Consider the subspace $|\mathscr{L}|=\bigcup_{\alpha \in \mathscr{L}} V_{\alpha} \subset \mathbf{C}^{n}$. In fact, it is a stratified set with the stratification induced by the multiple intersections of different spaces $V_{\alpha}$ parameterized by $\mathscr{L}$.

We say that the family $\mathscr{L}$ is totally real if any $V_{\alpha} \subset|\mathscr{L}|, \alpha \in \mathscr{L}$, is a totally real affine subspace of $\mathbf{C}^{n}$, i.e. it does not contain any complex line. Of course, if $\mathscr{L}$ is totally real, then its dimension $\operatorname{dim} \mathscr{L}=$ $\max _{\alpha \in \mathscr{L}}\left\{\operatorname{dim}_{\mathbf{R}} V_{\alpha}\right\}$ is not greater than $n$. 
We denote by $V_{\alpha}^{\mathbf{C}}$ the complexification of $V_{\alpha} \subset \mathbf{C}^{n}$ (which for totally real $V_{\alpha}$ is an affine subspace of real dimension $2 \operatorname{dim} V_{\alpha}$ ). We call a totally real family $\mathscr{L}$ weakly generic if the following holds: $V_{\beta} \nsupseteq V_{\alpha}$ implies $V_{\beta}^{\mathrm{C}} \nsupseteq V_{\alpha}$ for any $\alpha, \beta \in \mathscr{L}$.

One can associate a new family $D \mathscr{L}$ with any (totally real) family $\mathscr{L}$. This derived family $D \mathscr{L}$ is formed by all the spaces $V_{\alpha, \beta}=V_{\alpha} \cap V_{\beta}^{\mathbf{C}}$, $V_{\beta} \nsupseteq V_{\alpha}$ and which are maximal with respect to inclusion relations. In fact, $|D \mathscr{L}|$ contains $V_{\alpha} \cap V_{\beta}$ for any pair $\alpha, \beta \in \mathscr{L}$. If $\mathscr{L}$ is weakly generic then $\operatorname{dim} \mathscr{L}>\operatorname{dim} D \mathscr{L}$. Moreover, if $\mathscr{L}$ is totally real then $D \mathscr{L}$ also has this property.

We call a totally real family $\mathscr{L}$ perfectly generic if $\mathscr{L}$ and all its derived families $D \mathscr{L}, D(D \mathscr{L}), \ldots$, are weakly generic. Note, that if $\mathscr{L}$ is perfectly generic then its $(k+1)$-derivative $D^{(k+1)} \mathscr{L}=\varnothing$, where $k=\operatorname{dim} \mathscr{L}$.

The following Lemma is the main step to prove Theorem B.

LemMa 1. Given a totally real and perfectly generic family $\mathscr{L}$ of real affine subspace of $\mathbf{C}^{n}, \operatorname{dim} \mathscr{L}<n$, and any compact subset $K \subset|\mathscr{L}|$, then any continuous complex function on $K$ may be uniformly approximated by complex polynomials in coordinate functions $z_{1}, \ldots, z_{n}$ on $\mathbf{C}^{n}$. In particular, $K$ is polynomially convex in $\mathbf{C}^{n}$.

Let $C(K)$ be the algebra of all continuous functions on $K$. Let $\mathscr{P}(K)$ denote the uniform closure in $C(K)$ of the subalgebra multiplicatively generated by the functions $\operatorname{Res}_{K}\left(z_{i}\right), 1 \leq i \leq n$. By Bishop's theorem on maximal antisymmetric subdivisions to prove that $\mathscr{P}(K)=C(K)$ it is sufficient to show that any antisymmetry set $\Omega$ for $\mathscr{P}(K)$ is a singleton [3]. Recall, that a subset $\Omega \subseteq K$ is called an antisymmetry set for $\mathscr{P}(K)$ if any function $f \in \mathscr{P}(K)$ which is real valued on $\Omega$, in fact, is constant.

As a first step we prove that any antisymmetry set $\Omega$ is a singleton or is contained in the intersection of $K$ with the derived family $|D \mathscr{L}|$ (providing that $\mathscr{L}$ is totally real and weakly generic). Denote by $\Omega_{\alpha}$ the intersection $V_{\alpha} \cap \Omega$ and by $\Omega_{\alpha}$ the intersection $\dot{V}_{\alpha} \cap \Omega$, where $\stackrel{V}{V}_{\alpha}=$ $V_{\alpha} \backslash\left(V_{\alpha} \cap|D \mathscr{L}|\right)=V_{\alpha} \backslash \bigcup_{\beta \neq \alpha}\left(V_{\alpha} \cap V_{\beta}^{\mathrm{C}}\right)$. Note that $\mathscr{L}_{\text {weakly generic im- }}$ plies that $\dot{V}_{\alpha}$ is open and everywhere dense in $V_{\alpha}$.

For any two points $a, b \in \Omega_{\alpha}, \alpha \in \mathscr{L}$, we construct a polynomial $P_{\alpha}=P_{\alpha}\left(z_{1}, \ldots, z_{n}\right)$ which is real-valued on $|\mathscr{L}|$ and separates $a$ and $b$. Note, that for any two points $a, b \notin V_{\beta}^{\mathrm{C}}$ one can find a linear polynomial $L_{\beta}: \mathbf{C}^{n} \rightarrow \mathbf{C}$ which is zero on $V_{\beta}^{\mathbf{C}}$ and such that $L_{\beta}(a) \neq 0 \neq L_{\beta}(b)$. Now take the product $Q_{\alpha}=\prod_{\beta \neq \alpha} L_{\beta}$. The polynomial $Q_{\alpha}$ is zero on each $V_{\beta}$, $\beta \neq \alpha$, and $Q_{\alpha}(a) \neq 0 \neq Q_{\alpha}(b)$. Over $V_{\alpha}$ one can represent $Q_{\alpha}$ in the 
form $S_{\alpha}+i T_{\alpha}$ where the polynomials $S_{\alpha}: V_{\alpha} \rightarrow \mathrm{C}, T_{\alpha}: V_{\alpha} \rightarrow \mathrm{C}$ are real valued. Denote by $\tilde{Q}_{\alpha}^{*}: V_{\alpha} \rightarrow \mathrm{C}$ the polynomial $S_{\alpha}-i T_{\alpha}$. Using that $V_{\alpha}$ is totally real, one can extend $\tilde{Q}_{\alpha}^{*}$ to a polynomial $Q_{\alpha}^{*}: \mathbf{C}^{n} \rightarrow \mathbf{C}$ (first take the analytic extension of $\tilde{Q}_{\alpha}^{*}$ from $V_{\alpha}$ to $V_{\alpha}^{\mathbf{C}}$ and then use a complex linear projection $\mathbf{C}^{n} \rightarrow V_{\alpha}^{\mathbf{C}}$ ).

Consider the product $Q_{\alpha} Q_{\alpha}^{*}$ of the polynomials $Q_{\alpha}$ and $Q_{\alpha}^{*}$. This complex polynomial has the following remarkable properties: (1) $\left.Q_{\alpha} Q_{\alpha}^{*}\right|_{V_{\beta}}$ $\equiv 0$ for any $\beta \neq \alpha$; (2) $Q_{\alpha} Q_{\alpha}^{*}$ is real valued on $V_{\alpha}$; (3) $Q_{\alpha} Q_{\alpha}^{*}(a) \neq 0 \neq$ $Q_{\alpha} Q_{\alpha}^{*}(b)$.

Again, using that $V_{\alpha}$ is totally real, one can construct some polynomial $G_{\alpha}: \mathbf{C}^{n} \rightarrow \mathbf{C}$ which is real-valued on $V_{\alpha}$ and such that $G_{\alpha} Q_{\alpha} Q_{\alpha}^{*}(a)$ $\neq G_{\alpha} Q_{\alpha} Q_{\alpha}^{*}(b)$ (recall, that $Q_{\alpha} Q_{\alpha}^{*}$ cannot simultaneously vanish at $a$ and $b$ ). Hence, the polynomial $P_{\alpha}=G_{\alpha} \cdot Q_{\alpha} \cdot Q_{\alpha}^{*}$ separates $a$ and $b$. Moreover, it is real-valued on $V_{\alpha}$ and vanishes on any $V_{\beta}, \beta \neq \alpha$. Consequently, $\Omega_{\alpha}$ is a singleton or $\Omega_{\alpha} \subset|D \mathscr{L}|$. In fact, if $\Omega_{\alpha}=\Omega_{\alpha}$ is a singleton $a$, then $\Omega=\Omega_{\alpha}$ (note that $Q_{\alpha} Q_{\alpha}^{*}(a) \neq 0$ and, hence, it separates $a$ from $|D \mathscr{L}| \cup$ $\left(\cup_{\beta \neq \alpha} V_{\beta}\right) \subset \cup_{\beta \neq \alpha} V_{\beta}^{\mathrm{C}}$.

To complete the proof of Lemma 1 we apply inductively the same argument to the derived families $D \mathscr{L}, D^{2} \mathscr{L}, \ldots$ and use that $\mathscr{L}$ is perfectly generic (i.e. each $D^{s} \mathscr{L}, s=1,2, \ldots$ is weakly generic and totally real).

As we mentioned before, $|\mathscr{L}|$ is a stratified space with the stratification induced by different intersections $V_{\hat{\alpha}}=V_{\alpha_{1}} \cap V_{\alpha_{2}} \cap \cdots \cap V_{\alpha_{t}}, \alpha_{1}$, $\alpha_{2}, \ldots, \alpha_{t} \in \mathscr{L}$. In this way, starting with $\mathscr{L}$ one can produce a new family $\hat{\mathscr{L}} \supset \mathscr{L}$ of real affine subspaces parameterizing different multiple intersections. Let us say that $\mathscr{L}$ is a generic family if any two spaces $V_{\hat{\alpha}}$ and $V_{\hat{\beta}}^{\mathbf{C}}$ are in "general position" in $\mathbf{C}^{n}$ for each pair $\hat{\alpha}, \hat{\beta} \in \hat{\mathscr{L}}$, i.e. $V_{\hat{\alpha}} \cap V_{\hat{\beta}}^{\mathrm{C}}$ is of the smallest possible dimension, provided that $V_{\hat{\alpha}} \cap V_{\hat{\beta}}$ is fixed. More precisely, the spaces $W_{\hat{\alpha}, \hat{\beta}} \subseteq V_{\alpha}$ and $V_{\beta}^{C}$ should be in general position as real subspaces of $\mathbf{C}^{n}$, where $W_{\hat{\alpha}, \hat{\beta}}$ denotes a subspace of $V_{\hat{\alpha}}$ which does not intersect $V_{\hat{\alpha}} \cap V_{\hat{\beta}}$ and which is of a maximal possible dimension.

Denote by $\operatorname{LImb}\left(|\mathscr{L}|, \mathbf{C}^{n}\right)$ the space of all linear imbeddings of the space $|\mathscr{L}|$ into $\mathbf{C}^{n}$. Here $|\mathscr{L}|$ is considered without the ambient space $\mathbf{C}^{n}$, but with the fixed real linear structure for each $V_{\hat{\alpha}} \subset|\mathscr{L}|, \hat{\alpha} \in \hat{\mathscr{L}}$. Let $A(2 n, \mathbf{R})$ be the Lie group of all real affine transformations of $\mathbf{R}^{2 n} \simeq \mathbf{C}^{n}$. This group acts naturally on $\operatorname{LImb}\left(|\mathscr{L}|, \mathbf{C}^{n}\right)$. For any $F \in \operatorname{L} \operatorname{Imb}\left(|\mathscr{L}|, \mathbf{C}^{n}\right)$ and $g \in A(2 n, \mathbf{R})$ we denote by $g(F)$ the imbedding

$$
|\mathscr{L}| \stackrel{F}{\rightarrow} F(|\mathscr{L}|) \stackrel{g}{\rightarrow} g(F(|\mathscr{L}|)) \subset \mathbf{C}^{n}
$$


Lemma 2. Let $\operatorname{dim} \mathscr{L} \leq n$. Then the linear imbeddings $F:|\mathscr{L}| \rightarrow \mathbf{C}^{n}$ with the property " $F(|\mathscr{L}|)$ is totally real and generic" form an open and everywhere dense set $\mathscr{G}$ in the space $\mathrm{L} \operatorname{Imb}\left(|\mathscr{L}|, \mathbf{C}^{n}\right)$. Moreover, for any $F_{0} \in \mathrm{L} \operatorname{Imb}\left(|\mathscr{L}|, \mathbf{C}^{n}\right)$ the set $A_{F_{0}}$ of affine transformations $g$ with the property $g\left(F_{0}\right) \in \mathscr{G}$ form an open and everywhere dense subset of $A(2 n, \mathbf{R})$.

The properties of $F(|\mathscr{L}|)$ being totally real and generic are both general position properties. Hence, the openness of $\mathscr{G}$ in $\operatorname{L~} \operatorname{Imb}\left(|\mathscr{L}|, \mathrm{C}^{n}\right)$ or of $A_{F_{0}}$ in $A(2 n, \mathbf{R})$ is obvious. So, we have to prove that $\mathscr{G}$ and $A_{F_{0}}$ are everywhere dense in the corresponding spaces.

For any $V_{\alpha} \subset F_{0}(|\mathscr{L}|), \alpha \in \mathscr{L}, F_{0} \in \mathrm{L} \operatorname{Imb}\left(|\mathscr{L}|, \mathbf{C}^{n}\right)$ consider the subset $\rho_{\alpha} \subset A(2 n, \mathbf{R})$ such that $g \in \rho_{\alpha}$ iff $g\left(V_{\alpha}\right)$ is totally real. If $\operatorname{dim} V_{\alpha} \leq n$ then one can check that $\rho_{\alpha}$ is open and everywhere dense in $A(2 n, \mathbf{R})$. Consequently, $\rho_{\mathscr{L}}=\bigcap_{\alpha \in \mathscr{L}} \rho_{\alpha}$ is open and everywhere dense as well. Picking some $\tilde{g} \in \rho_{\mathscr{L}}$ sufficiently close to the identity one can approximate $F_{0}$ by a totally real imbedding $\tilde{F}_{0}=\tilde{g}\left(F_{0}\right)$. Hence, for $\operatorname{dim} \mathscr{L} \leq n$ totally real imbeddings are everywhere dense in $\operatorname{LImb}\left(|\mathscr{L}|, \mathbf{C}^{n}\right)$. Now take any pair of affine subspaces $V_{\hat{\alpha}}, V_{\hat{\beta}} \subset \tilde{F}_{0}(|\mathscr{L}|), \hat{\alpha}, \hat{\beta} \in \hat{\mathscr{L}}$, such that $V_{\hat{\alpha}} \varsubsetneqq V_{\hat{\beta}}$. Recall, that $W_{\hat{\alpha}, \hat{\beta}}$ is a subspace of $V_{\hat{\alpha}}$ of a maximal dimension such that $W_{\hat{\alpha}, \hat{\beta}} \cap$ $\left(V_{\hat{\alpha}} \cap V_{\hat{\beta}}\right)=\varnothing$. Consider the following subset $\Sigma_{\hat{\alpha}, \hat{\beta}} \subset A(2 n, \mathbf{R})$. An element $g \in \Sigma_{\hat{\alpha}, \hat{\beta}}$ iff $g\left(W_{\hat{\alpha}, \hat{\beta}}\right)$ is in general position with the complex subspace $\left[g\left(V_{\hat{\beta}}\right)\right]^{\mathrm{C}}$. Again, the openness of $\Sigma_{\hat{\alpha}, \hat{\beta}}$ is obvious. To prove that $\Sigma_{\hat{\alpha}, \hat{\beta}}$ is dense in $A(2 n, \mathbf{R})$ we show that the identity transformation $e \in A(2 n, \mathbf{R})$ can be approximated by some $g \in A(2 n, \mathbf{R})$ with the property $g\left(V_{\hat{\beta}}\right)=V_{\hat{\beta}}$ and $g\left(W_{\hat{\alpha}, \hat{\beta}}\right)$ being transversal to $V_{\beta}^{\mathbf{C}}$. Note, that by the construction, $W_{\hat{\alpha}, \hat{\beta}}$ and $V_{\hat{\beta}}$ are in general position in $\mathbf{C}^{n}$. Take $\tilde{W}_{\hat{\alpha}, \hat{\beta}} \subset \mathbf{C}^{n}$ sufficiently close to $W_{\hat{\alpha}, \hat{\beta}}$ (so it still will be in general position with $V_{\hat{\beta}}$ ) and transverse to $V_{\hat{\beta}}^{\mathbf{C}}$. Now it is easy to construct a real affine transformation $g$ mapping $W_{\hat{\alpha}, \hat{\beta}}$ onto $\tilde{W}_{\hat{\alpha}, \hat{\beta}}$ and identical on $V_{\hat{\beta}}$. Moreover, this $g$ can be taken close to $e$. So, the subset $\Sigma_{\hat{\mathscr{L}}}=\rho_{\mathscr{L}} \cap\left(\bigcap_{\left\{v_{\hat{\alpha} \subsetneq} \subsetneq V_{\hat{\beta}}\right\}} \Sigma_{\hat{\alpha}, \hat{\beta}}\right)$ of $A(2 n, \mathbf{R})$ is open and everywhere dense. This implies that totally real and generic imbeddings are open and everywhere dense in $\operatorname{L} \operatorname{Imb}\left(|\mathscr{L}|, \mathbf{C}^{n}\right)$, provided that $\operatorname{dim} \mathscr{L} \leq n . \square$

LEMMA 3. If $\operatorname{dim} \mathscr{L} \leq \frac{2}{3} n$ then $\mathscr{L}$ totally real and generic implies that $\mathscr{L}$ is perfectly generic. Consequently, the set of imbeddings $F \in \operatorname{L} \operatorname{Imb}\left(|\mathscr{L}|, \mathbf{C}^{n}\right)$ with the property " $\mathscr{P}(K)=C(K)$ " for any compact $K \subset F(|\mathscr{L}|)$ contains an open and everywhere dense subset of $\mathrm{L} \operatorname{Imb}\left(|\mathscr{L}|, \mathbf{C}^{n}\right)$.

If $\operatorname{dim} V_{\hat{\alpha}}+2 \operatorname{dim} V_{\hat{\beta}} \leq 2 n ; \hat{\alpha}, \hat{\beta} \in \hat{\mathscr{L}}$, and $\mathscr{L}$ is generic then $V_{\hat{\alpha}} \cap V_{\hat{\beta}}^{\mathbf{C}}$ $=V_{\hat{\alpha}} \cap V_{\hat{\beta}}$ (when $V_{\hat{\alpha}} \cap V_{\hat{\beta}} \neq \varnothing$ ) or $V_{\hat{\alpha}} \cap V_{\hat{\beta}}^{\mathrm{C}}$ is at most a singleton (when $V_{\hat{\alpha}} \cap V_{\hat{\beta}}=\varnothing$ and $\operatorname{dim} V_{\hat{\alpha}}+2 \operatorname{dim} V_{\hat{\beta}}=2 n$ ) (see Fig. 1). Hence, under 
these dimensional assumptions $|D \mathscr{L}|=\left|\mathscr{L}^{\prime}\right| \cup M$, where $\left|\mathscr{L}^{\prime}\right|$ is formed by $V_{\hat{\alpha}}, \hat{\alpha} \in \hat{\mathscr{L}} \backslash \mathscr{L}$ (i.e. $\hat{\alpha}$ is not a maximal element of $\hat{\mathscr{L}}$ ) and $M$ is a finite set of points (0-dimensional subspaces) in $\mathbf{C}^{n}$. Note, that $\mathscr{L}$ generic implies that $\mathscr{L}^{\prime} \cup M$ is a generic family too. In fact, any subfamily of a generic family is generic. So, $\mathscr{L}^{\prime}$ is generic. By the construction $V_{\hat{\beta}}^{\mathbf{c}} \cap M$ $=\varnothing$ for any $\hat{\beta} \in \hat{\mathscr{L}}^{\prime}$. All the higher derivatives $D^{s} \mathscr{L}, s>1$, will be just subfamilies of $\hat{\mathscr{L}}$ and, hence, are generic (weakly generic). So, $\mathscr{L}$ is perfectly generic and Lemmas 1 and 2 imply Lemma 3.

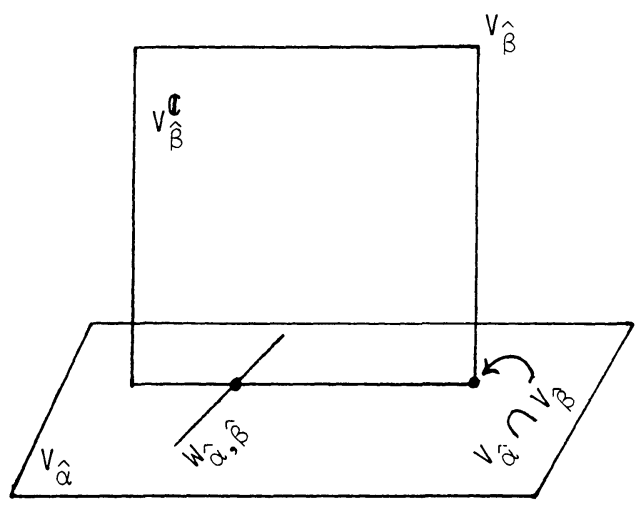

FIGURE 1

REMARK. Lemma 3 is the only place where we are using the dimensional restriction $\operatorname{dim} X \leq \frac{2}{3} n$. We conjecture that this lemma holds just if $\operatorname{dim} \mathscr{L}<n$, which would imply Theorems A and B for compact spaces or for finite polyhedra of dimensions less than $n$.

Now we are able to prove Theorem B. Any simplicially linear mapping $F: Y^{k} \rightarrow \mathbf{C}^{n}$ is uniquely determined by the images $\left\{F\left(y_{j}\right)\right\}_{j}$ of the vertices $\left\{y_{j}\right\}_{j}$ of the simplicial polyhedron $Y^{k}$. If the points $\left\{F\left(y_{j}\right)\right\}$ are in general position over the field $\mathbf{R}$ in $\mathbf{C}^{n} \simeq \mathbf{R}^{2 n}$, it follows from standard dimensional considerations that $F$ is an imbedding for $k<n$. Actually, if they are in general position in $\mathbf{C}^{n}$ over $\mathbf{C}$, then any real affine subspace passing through arbitrary $s$ points $\left\{F\left(y_{j_{e}}\right)\right\}_{e}, s \leq n$, is totally real.

Let $\Delta_{\alpha}^{s} \subset Y^{k}$ denote an $s$-dimensional simplex of $Y^{k}$, where index $\alpha$ enumerates such simplices. For any $\Delta_{\alpha}^{s} \subset Y^{k}$ and $F \in \operatorname{SL} \operatorname{Imb}\left(Y^{k}, \mathbf{C}^{n}\right)$ consider the real $s$-dimensional affine subspace $V_{\alpha, F}$ in $\mathbf{C}^{n}$, containing $F\left(\Delta_{\alpha}^{s}\right)$. This correspondence $\Delta_{\alpha}^{s} \leadsto V_{\alpha, F}$ defines a family of subspaces $\hat{\mathscr{L}}_{F}$ (the corresponding family $\mathscr{L}_{F}$ consists of $V_{\alpha, F}$, where $\Delta_{\alpha}^{s}$ is not a subsimplex of any other simplex of $Y^{k}$ ). 
Starting with any mapping $F \in \operatorname{SLMap}\left(Y^{k}, \mathbf{C}^{n}\right)$ one can approximate $F$ by an imbedding $\tilde{F}(k<n)$. Note that the group $A(2 n, \mathbf{R})$ acts naturally on $\operatorname{SLMap}\left(Y^{k}, \mathbf{C}^{n}\right)$, moreover, the subspace $\operatorname{SLImb}\left(Y^{k}, \mathbf{C}^{n}\right) \subset$ $\operatorname{SL} \operatorname{Map}\left(Y^{k}, \mathbf{C}^{n}\right)$ obviously is invariant under this action. By Lemma 2 and using the continuity of the correspondence $F \leadsto\left|\mathscr{L}_{F}\right|$ one can approximate $\tilde{F} \in \operatorname{SL} \operatorname{Imb}\left(Y^{k}, \mathbf{C}^{n}\right)$ by some imbedding $g(\tilde{F}), g \in A(2 n, \mathbf{R})$ with the property $g\left(\left|\mathscr{L}_{\tilde{F}}\right|\right)$ is totally real and generic. By Lemma 3 such a family will be perfectly generic, provided that $3 k \leq 2 n$. Hence, by Lemma $1 g\left(\tilde{F}\left(Y^{k}\right)\right) \subset g\left(\left|\mathscr{L}_{\tilde{F}}\right|\right)$ admits polynomial approximation.

The properties " $\mathscr{L}_{F}$ totally real, generic, perfectly generic" obviously are stable with respect to small perturbations of $F \in \operatorname{SLImb}\left(Y^{k}, \mathbf{C}^{n}\right)$. Hence, for $k \leq \frac{2}{3} n$ the subset $\left\{F \in \operatorname{SL} \operatorname{Imb}\left(Y^{k}, \mathbf{C}^{n}\right) \mid \mathscr{L}_{F}\right.$ is totally real and perfectly generic $\}$ is open and everywhere dense in $\operatorname{SL} \operatorname{Map}\left(Y^{k}, \mathbf{C}\right)$, which completes the proof of Theorem B.

Now we derive Theorem A from Theorem B.

Let $X^{k}$ be any compact space. Let $\Theta_{\varepsilon, \delta}$ be the subset of $\operatorname{Map}\left(X^{k}, \mathbf{C}^{n}\right)$ defined by the following two properties: (1) the diameter of the inverseimage $F^{-1}(y)$ of any point $y \in \mathbf{C}^{n}$ is less than $\delta$; (2) the functions $\bar{z}_{1}, \ldots, \bar{z}_{n}$ on $F\left(X^{k}\right)$, where - denotes the complex conjugation, may be approximated to within $\varepsilon$ by complex polynomials in $z_{1}, \ldots, z_{n}$. It is readily verified that $\Theta_{\varepsilon, \delta}$ is an open set of $\operatorname{Map}\left(X^{k}, \mathbf{C}^{n}\right)$.

Now choose some countable monotone sequence $\left\{\varepsilon_{i}\right\} \rightarrow 0,\left\{\delta_{i}\right\} \rightarrow 0$. It is easy to verify that $\bigcap_{i} \Theta_{\varepsilon_{i}, \delta_{i}}$ is the set $\Theta$ of all imbeddings $F$ admitting polynomial approximation on $F\left(X^{k}\right)$. Indeed, if we let $\delta_{i} \rightarrow 0$ property (1) of the sets $\Theta_{\varepsilon_{t}, \delta_{t}}$ guarantees that the limiting mapping is an imbedding. Property (2) of the sets implies that if $F \in \bigcap_{l} \Theta_{\varepsilon_{l}, \delta_{t}}$ then the functions $\left\{\bar{z}_{j}\right\}$ on the image $F\left(X^{k}\right)$ may be approximated to within arbitrary accuracy by polynomials in $\left\{z_{j}\right\}$. On the other hand, by the Weierstrass-Stone theorem any continuous function on $F\left(X^{k}\right)$ may be approximated by polynomials in $\left\{z_{j}, \bar{z}_{j}\right\}$; hence it may be approximated by polynomials in the variables in the variables $\left\{z_{j}\right\}$ alone.

To complete the proof, it remains to verify that every set $\Theta_{\varepsilon_{t}, \delta_{t}}$ is dense in $\operatorname{Map}\left(X^{k}, \mathbf{C}^{n}\right)$.

Let $F^{\prime} \in \operatorname{Map}\left(X^{k}, \mathbf{C}^{n}\right)$ be an arbitrary mapping. In accordance with the classical Alexandroff construction [1], if $m<n$, then for any $\varepsilon, \delta>0$ there is a mapping $F: X^{k} \rightarrow \mathbf{C}^{n}$ such that $F\left(X^{k}\right)$ is contained in a $k$-dimensional simplicial polyhedron $Y^{k}$ simplicially-linearly imbedded in $\mathbf{C}^{n}$, in such a way that

(a) $\rho\left(F^{\prime}, F\right)<\varepsilon$, where $\rho$ is the natural distance between mappings;

(b) $\operatorname{diam}\left(F^{-1}(y)\right)<\delta$ for any point $y \in Y^{k}$. 
(A complete proof of this theorem can also be found in [4], Chapter V, §3).

Set $\delta=\delta_{i}$. By a trivial modification of this construction one can guarantee that, in addition to these two properties (a) and (b), the family of affine subspaces $\mathscr{L}_{\text {id }}$ (generated by id: $Y^{k} \rightarrow \mathbf{C}^{n}$ ) will be totally real and generic (just use the appropriate transformation from $A(2 n, \mathbf{R})$ ). If $3 k \leq 2 n$ then, by Lemma 3 , these properties are a sufficient condition for the existence of polynomial approximation on the polyhedron $Y^{k}$. The modification is as follows. By Theorem B there exists an imbedding $\kappa$ : $Y^{k} \rightarrow \mathbf{C}^{n}$, arbitrarily close to the original imbedding id: $Y^{k} \rightarrow \mathbf{C}^{n}$, such that continuous functions admit polynomial approximation on $\kappa\left(Y^{k}\right)$. The imbedding $\kappa \in \operatorname{SL} \operatorname{Map}\left(Y^{k}, \mathbf{C}^{n}\right)$ may be chosen in such a way that $\rho\left(F^{\prime}, \kappa \circ F\right)<\varepsilon$, while $\operatorname{diam}\left(F^{-1} \circ \kappa^{-1}(y)\right)<\delta_{t}$ for any $y \in \mathbf{C}^{n}$. Moreover, the functions $\left\{\bar{z}_{j}\right\}$ may be approximated on $\kappa \circ F\left(X^{k}\right)$ to within arbitrary accuracy by polynomials in $\left\{z_{i}\right\}$, i.e., $\kappa \circ F \in \Theta_{\varepsilon_{t}, \delta_{t}}$ and $\kappa \circ F$ is in the $\varepsilon$-neighborhood of the original mapping $F^{\prime}$. This proves that $\Theta_{\varepsilon_{t}, \delta_{l}}$ is dense in $\operatorname{Map}\left(X^{k}, \mathbf{C}^{n}\right)$.

Recall that for any compact set $K$ in $\mathbf{C}^{n}$ the space of maximal ideals of the algebra $\mathscr{P}(K)$ is precisely the polynomially convex hull of $K$. Therefore, if $\mathscr{P}(K)$ coincides with the algebra of all complex functions, then $K$ is polynomially convex and this property is hereditary with respect to compact subsets of $K$. Thus, if $3 k \leq 2 n$ the polynomially convex imbeddings of a $k$-dimensional compact space into $\mathbf{C}^{n}$ form a massive set (i.e. of type $G_{\delta}$ ). This completes the proof of Theorem A.

It is obvious that if all continuous functions on a compact subset $K \subset \mathbf{C}^{n}$ admit polynomial approximation, this property is hereditary with respect to closed subsets and therefore, in particular, the intersection $K \cap \mathbf{C}^{l}$ of a compact subset $K$ with any affine complex subspace also admits approximation by polynomials in $z_{1}, \ldots, z_{n}$. In particular, in the case $k=l$, it follows from the maximum modulus theorem that the set $K \cap \mathbf{C}^{1}$ is necessarily nowhere dense in $\mathbf{C}^{1}$ and has connected complement.

COROLlary. Let $X^{k}$ be a $k$-dimensional compact space. If $3 k \leq 2 n$, the imbeddings $F \in \operatorname{Map}\left(X^{k}, \mathbf{C}^{n}\right)$ such that the intersection of $F\left(X^{k}\right)$ with any complex straight line $\mathbf{C}^{1} \subset \mathbf{C}^{n}$ is nowhere dense in $\mathbf{C}^{1}$ and the complement of the intersection is connected in $\mathbf{C}^{1}$ form a dense subset of type $G_{\delta}$.

Let $M^{k}$ be a PL-manifold. Then starting with an arbitrary locally flat PL-imbedding $F_{0}: M^{k} \rightarrow \mathbf{C}^{n}(k<n)$ it is possible to find an element $g \in A(2 n, \mathbf{R})$ such that $g\left(F_{0}\right)\left(M^{k}\right)$ will generate a totally real and generic 
family of affine subspaces and, hence, for $k \leq \frac{2}{3} n$ one has polynomial approximation on $g\left(F_{0}\right)\left(M^{k}\right)$. Moreover, $g\left(F_{0}\right)\left(M^{k}\right)$ is again locally flat. Thus, by Theorem B for $k \leq \frac{2}{3} n$ there exists a PL-imbedding $F$ of $M^{k}$ in $\mathrm{C}^{n}$ with $F\left(M^{k}\right)$ having a nice normal $P L$-bundle and admitting polynomial approximation (hence, $F\left(M^{k}\right)$ is polynomially convex in $\mathbf{C}^{n}$ ). In particular, the tangent bundle to $F\left(M^{k}\right)$ is formed by "totally real" blocks.

Considering smooth or real-analytic manifolds $M^{k}$, it would be natural to try to prove "smooth or analytic" analogs of Theorems A and B. But it seems quite unlikely that such propositions can be established. As a matter of fact, for $k \geq \frac{2}{3} n$ there exist profound topological obstacles to the existence of totally real and regular imbedding, i.e., imbeddings $F: M^{k} \rightarrow$ $\mathrm{C}^{n}$ such that $d F$ is nondegenerate and $d F\left(T_{x} M^{k}\right)$ is totally real for any tangent space $T_{x} M^{k}$ of $M^{k}, x \in M^{k}$.

One can find a very good discussion of similar and more delicate analytic phenomena in [7] and [8] $\S 17,18$ bascially, for the case $k \geq n$.

As an example, let us consider regular imbeddings $F: \mathbf{C} P^{k} \rightarrow \mathbf{C}^{n}$ of complex projective space $\mathbf{C} P^{k}$. Let $\tau$ be a tangent bundle of $F\left(\mathbf{C} P^{k}\right)$ and assume that it is a totally real subbundle of the complex tangent bundle to $\mathbf{C}^{n}$. Hence, its complexification $\tau^{\mathbf{C}}$ is isomorphic to $\tau \oplus J \tau$, where the infinitesimal operator $J$ is induced by multiplication of vectors by the imaginary unit $i$. Let $\nu$ be the bundle complementary to $\tau \oplus J(\tau)$, i.e., $\tau \oplus J(\tau) \oplus \nu=\tau\left(\mathbf{C}^{n}\right) \mid F\left(\mathbf{C} P^{k}\right)$ is the trivial bundle. Since $\tau_{x} \oplus J\left(\tau_{x}\right)$ is a complex subspace of $\mathbf{C}^{n}$, we may assume that $\nu$ is a complex bundle of complex dimension $n-2 k$. The Chern class $c\left(\tau^{\mathbf{C}}\right)$ of $\tau^{\mathbf{C}}$ is equal to

$$
\sum_{i=0}^{k} c_{i}(\tau) \times \sum_{i=0}^{k}(-1)^{i} c_{i}(\tau) \text { or }\left(1-h^{2}\right)^{k+1}
$$

where $h \in H^{2}\left(\mathbf{C} P^{k} ; Z\right)$ is a standard generator and $\left(1-h^{2}\right)^{k+1}$ is considered as an element of the ring $\mathbf{Z}[h] /\left\{h^{k+1}=0\right\}$ [5]. Since $\tau^{C} \oplus \nu$ is trivial, it follows that $c(\nu) \cdot c\left(\tau^{\mathbf{C}}\right)=1$. The element $c\left(\tau^{\mathbf{C}}\right)$ is invertible in the ring $\mathbf{Z}[h] /\left\{h^{k+1}=0\right\}$. As a representative of the inverse element, we take the polynomial $\left[\sum_{i=0}^{[k / 2]} h^{2 l}\right]^{k+1}$, where $[k / 2]$ is the integral part of $k / 2$. After factorization modulo $h^{k+1}=0$ we obtain a certain polynomial $\sum_{i=0}^{[k / 2]} \alpha_{i} h^{2 i}$, where $\left\{\alpha_{i}\right\}$ are different from zero. Therefore $c(\nu)=$ $\sum_{i=0}^{[k / 2]} \alpha_{i} h^{2 i}$ and, since $\alpha_{i} \neq 0$, the complex dimension $n-2 k$ of $\nu$ cannot be less than $2 \cdot[k / 2]$. Thus, when $n<2 k+2[k / 2]$, there exist no totally real immersions of $\mathbf{C} P^{k}$ into $\mathbf{C}^{n}$. In fact, the Euler class of the normal bundle of oriented submanifolds in $\mathbf{R}^{2 n}$ should be trivial [5], which ruins the possibility for regular totally real imbeddings $\mathbf{C} P^{k} \hookrightarrow \mathbf{C}^{3 k}, k$ even. Since $\operatorname{dim}_{\mathbf{R}} \mathbf{C} P^{k}=2 k$, it follows that the "allowed" dimensions $n$ satisfy 
the conditions $3 \operatorname{dim}_{\mathbf{R}}\left(\mathbf{C} P^{k}\right)<2 n$, which should be compared with the dimensional condition that figures in Theorems $\mathrm{A}$ and $\mathrm{B}$.

\section{REFERENCES}

[1] P. Alexandrov and B. Passinkov, Introduction to dimension theory, Nauka, Moscow 1973.

[2] A. Browder, Cohomology of maximal ideal spaces, Bull. A.M.S., 67 (1961), 515-516.

[3] W. Gamelin, Uniform Algebras, Prentice-Hall, Inc., Englewood Cliffs, NJ 1969.

[4] W. Hurewicz and H. Wallman, Dimension Theory, Princeton University Press, 1948.

[5] J. W. Milnor and J. D. Stasheff, Characteristic Classes, Annals of Math. Studies, Princeton University Press 1974.

[6] D. Vodovoz and M. Zeidenberg, On the number of generators of the algebra of continuous functions, Mathematicheskie Zametki (USSR), 10, issue 5, (1971), 537-540.

[7] R. O. Wells, Jr., Function Theory on Differentiable Submanifolds, Contributions to Analysis, Academic Press, New York-London 1974, 407-441.

[8] J. Wermer, Banach Algebras and Several Complex Variables, Springer-Verlag, New York, Heidelberg, Berlin 1976.

[9] H. Whitney, On the topology of differentiable manifolds, Lectures in Topology, Univ. of Michigan Press, 1941, 101-141.

Received June 28, 1984; in revised form February 3, 1985.

BEN-GURION UNIVERSITY OF NEGEV

BEER - SHEVA, 84 105, ISRAEL 


\section{PACIFIC JOURNAL OF MATHEMATICS EDITORS}

V. S. VARADARAJAN (Managing Editor)
University of California
Los Angeles, CA 90024
HEBERT CLEMENS
University of Utah
Salt Lake City, UT 84112
CHARLES R. DEPRIMA
California Institute of Technology
Pasadena, CA 91125

\section{R. FINN}

Stanford University

Stanford, CA 94305

HeRmanN FlaschKa

University of Arizona

Tucson, AZ 85721

RAMESH A. GANGOLli

University of Washington

Seattle, WA 98195

ROBION KIRBY

University of California

Berkeley, CA 94720

\section{C. MoOre}

University of California

Berkeley, CA 94720

H. SAMELSON

Stanford University

Stanford, CA 94305

HAROLD STARK

University of California, San Diego

La Jolla, CA 92093

ASSOCIATE EDITORS
R. ARENS
E. F. BECKENBACH
B. H. NeUmanN
F. WOLF
K. Yoshida (1906-1982)

\section{SUPPORTING INSTITUTIONS}

UNIVERSITY OF ARIZONA

UNIVERSITY OF BRITISH COLUMBIA

CALIFORNIA INSTITUTE OF TECHNOLOGY

UNIVERSITY OF CALIFORNIA

MONTANA STATE UNIVERSITY

UNIVERSITY OF NEVADA, RENO

NEW MEXICO STATE UNIVERSITY

OREGON STATE UNIVERSITY
UNIVERSITY OF OREGON

UNIVERSITY OF SOUTHERN CALIFORNIA

STANFORD UNIVERSITY

UNIVERSITY OF HAWAII

UNIVERSITY OF TOKYO

UNIVERSITY OF UTAH

WASHINGTON STATE UNIVERSITY

UNIVERSITY OF WASHINGTON 


\section{Pacific Journal of Mathematics}

\section{Vol. 121, No. $1 \quad$ November, 1986}

Om P. Agrawal, Douglas Napier Clark and Ronald George Douglas,

Invariant subspaces in the polydisk $\ldots \ldots \ldots \ldots \ldots \ldots \ldots \ldots \ldots \ldots$

Christoph Bandt and Gebreselassie Baraki, Metrically invariant measures

on locally homogeneous spaces and hyperspaces $\ldots \ldots \ldots \ldots \ldots \ldots \ldots$

Marcy Mason Barge, Horseshoe maps and inverse limits ..............29

Russell Gene Bilyeu, Robert Richard Kallman and Paul Weldon Lewis,

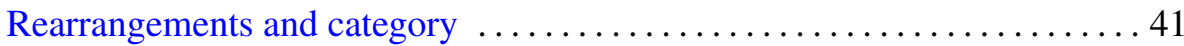

Jean Bourgain, A problem of Douglas and Rudin on factorization . . .......47

Hernan Cendra, A normal form and integration in finite terms for a class of

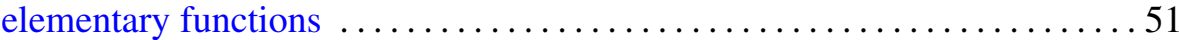

Ky Fan, The angular derivative of an operator-valued analytic function . . . . 67

Gerhard Gierz, On the Dunford-Pettis property of function modules of

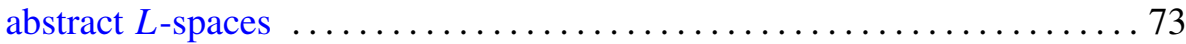

Gabriel Katz, On polynomial generators in the algebra of complex functions

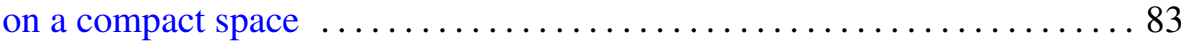

Ridgley Lange, Duality and asymptotic spectral decompositions $\ldots \ldots \ldots . .93$

Anthony To-Ming Lau and Peter F. Mah, Quasinormal structures for certain spaces of operators on a Hilbert space ................... 109

R. Daniel Mauldin, Correction: "The set of continuous nowhere differentiable functions"

Alan Harvey Mekler and Saharon Shelah, $\omega$-elongations and Crawley's problem

Alan Harvey Mekler and Saharon Shelah, The solution to Crawley's problem

Richard Rochberg, Deformation of uniform algebras on Riemann surfaces

Joseph Roitberg, On weak epimorphisms in homotopy theory

Jesús M. Ruiz, A remark on fields with the dense orbits property

Henry Wente, Counterexample to a conjecture of H. Hopf

David G. Wright, Rigid sets in $E^{n}$ 